University of Wollongong

Research Online

Faculty of Engineering and Information

Faculty of Engineering and Information

Sciences - Papers: Part B

Sciences

2018

\title{
A closed-form pricing formula for European options under the Heston model with stochastic interest rate
}

\author{
Xinjiang He \\ University of Wollongong, xinjiang@uow.edu.au \\ Song-Ping Zhu \\ University of Wollongong, spz@uow.edu.au
}

Follow this and additional works at: https://ro.uow.edu.au/eispapers1

Part of the Engineering Commons, and the Science and Technology Studies Commons

Research Online is the open access institutional repository for the University of Wollongong. For further information contact the UOW Library: research-pubs@uow.edu.au 


\title{
A closed-form pricing formula for European options under the Heston model with stochastic interest rate
}

\author{
Abstract \\ In this paper, a closed-form pricing formula for European options in the form of an infinite series is derived \\ under the Heston model with the interest rate being another random variable following the CIR (Cox- \\ Ingersoll-Ross) model. One of the main advantages for the newly derived series solution is that we can \\ provide a radius of convergence, which is complemented by some numerical experiments demonstrating \\ its speed of convergence. To further verify our formula, option prices calculated through our formula are \\ also compared with those obtained from Monte Carlo simulations. Finally, a set of pricing formulae are \\ derived with the series expanded at different points so that the entire time horizon can be covered by \\ converged solutions.

\section{Disciplines} \\ Engineering | Science and Technology Studies

\section{Publication Details} \\ He, X. \& Zhu, S. (2018). A closed-form pricing formula for European options under the Heston model with \\ stochastic interest rate. Journal of Computational and Applied Mathematics, 335 323-333.
}




\title{
A closed-form pricing formula for European options under the Heston model with stochastic interest rate
}

\author{
Xin-Jiang HE ${ }^{*} \quad$ Song-Ping ZHU ${ }^{\dagger}$
}

\begin{abstract}
In this paper, a closed-form pricing formula for European options in the form of an infinite series is derived under the Heston model with the interest rate being another random variable following the CIR (Cox-Ingersoll-Ross) model. One of the main advantages for the newly derived series solution is that we can provide a radius of convergence, which is complemented by some numerical experiments demonstrating its speed of convergence. To further verify our formula, option prices calculated through our formula are also compared with those obtained from Monte Carlo simulations. Finally, a set of pricing formulae are derived with the series expanded at different points so that the entire time horizon can be covered by converged solutions.
\end{abstract}

\section{AMS(MOS) subject classification.}

Keywords. European option, Series solution, Stochastic interest rate, Convergence.

\footnotetext{
* Corresponding author. School of Mathematics and Applied Statistics, University of Wollongong NSW 2522, Australia

${ }^{\dagger}$ School of Mathematics and Applied Statistics, University of Wollongong NSW 2522, Australia.
} 


\section{Introduction}

In 1973, Black \& Scholes [5] made a breakthrough by proposing an elegant model with the underlying price following a geometric Brownian motion and deriving an analytical formula for European option prices. However, some of its simplified assumptions made to achieve the analytical simplicity and tractability are inappropriate and can cause mis-pricing problems. In particular, one of its main drawbacks is the constant volatility assumption, which contradicts to the phenomenon of "volatility smile" [8] observed in real markets that the implied volatility extracted from real market data tends to exhibit a "smile" curve. As a result, a number of modifications have been proposed to incorporate the non-constant volatility into the Black-Scholes model.

Non-constant volatility models can mainly be divided into two categories, i.e., local volatility and stochastic volatility. The former, assuming that the volatility be a deterministic function of the underlying price and time, is considered by Dupire [9], Derman \& Kani [7] and Rubinstein [21]. Unfortunately, empirical studies have already suggested that

the "smile dynamics" are poorly captured by local volatility models (e.g., Hagan et. al [13]). Therefore, the latter category, making the volatility of the underlying price another random variable has thus become much more popular.

However, due to the addition of another stochastic source, it is very difficult to derive analytical solutions for most of stochastic volatility models, and numerical methods must be resorted to in these cases. For example, Johnson [19] and Scott [23] directly simulated the stochastic processes with the Monte Carlo simulation technique, while Wiggins [25] adopted the finite difference method to solve the PDEs (partial differential equations) governing option prices. Unfortunately, a noticeable pity for numerical methods is always the lack of speed in computation, which makes it difficult to implement these models in real markets since model calibration is very time-consuming and the lack of analytical pricing formula can make the situation even worse. Therefore, further research interest was led to 
finding more appropriate stochastic volatility processes with analytic pricing formula for European call options. Specifically, Hull \& White [17] proposed that the volatility follow another geometric Brownian motion and derived a power series solution for option prices. Albeit appealing, the assumption of the zero correlation between the underlying price and the volatility made in their model is at odds with the so-called "leverage effects" that the underlying price and the volatility should be negatively correlated [2]. Moreover, although an Ornstein-Uhlenbeck process is adopted for the volatility process in the Stein-Stein model [24] and a closed-form pricing formula is presented, some of the model flaws, such as unable to prevent the volatility from going negative, make this model still unsatisfactory. In 1993, Heston [16] contributed a lot to the literature by incorporating the CIR (CoxIngersoll-Ross) model to describe the volatility process and deriving a closed-form pricing formula for European options. Two main reasons can account for its popularity; one is that the volatility process itself satisfies a wide range of basic properties, such as the obvious non-negative property and the mean-reverting property being consistent with the results of empirical studies [3], and another is that there exists a closed-form formula when pricing options, which can save us a lot of time and effort when conducting model calibration. Given the fact that the introduction of the stochastic volatility makes the markets incomplete and there exist different equivalent martingale measures, the option price under the Heston model is not unique, and the analytical pricing formula derived by Heston can no longer be used if a different martingale measure is chosen. Recently, He \& Zhu $[15,26]$ presented a different analytical pricing formula for European options under the Heston model by choosing the so-called minimal entropy martingale measure.

However, it should also be pointed out that the well-known Heston model is not perfect either in many senses (there may not even be a perfect one!) and many attempts are made to improve its pricing performance in real markets, such as the introduction of the time-dependent Heston models [10] and the regime-switching Heston models [14]. One of the most popular approaches is to incorporate the stochastic interest rate into stochastic 
volatility models to form a hybrid model since there are a lot of empirical evidence suggesting that introducing stochastic interest rate into option pricing models can lead to better model performance $[1,20]$, and a number of authors have worked on this area. For instance, a combination of the correlated Stein-Stein model [22] and the Hull-White interest rate model [18] is adopted in [12] with European options evaluated under the Fourier cosine expansion framework. Furthermore, approximation formulae for European option prices are presented when the underlying price follows the Heston stochastic volatility model with the interest rate described by the CIR model [11].

In this paper, we adopt the Heston-CIR hybrid model for the underlying price and we aim to present a closed-form pricing formula for European options as models with exact and analytical solutions are much more favored in real markets. Based on the technique of numeraire change, we firstly obtain a general pricing formula with the unknown characteristic function of the underlying price under a forward measure. Then, the target characteristic function is analytically worked out written in the form of an infinite series by expending the solution in terms of the time to expiry; such a series solution is accompanied by a proof of convergence that a radius of convergence is theoretically figured out. Numerical experiments are carried out to show the the convergence speed as well as the accuracy of the newly derived formula. Finally, for the situation that the time to expiry is larger than the provided radius of convergence, we have also come out an alternative way by deriving a set of pricing formulae converging on a particular region with different expansion points so that the entire time horizon can be covered by converged solutions.

The rest of the paper is organized as follows. In Section 2, a brief introduction of the Heston-CIR hybrid model is provided. In Section 3, we firstly introduce a general pricing approach, after which we present an analytical pricing formula in a series form based on the change of numeraire and the derivation of the characteristic function. A radius of convergence for this series solution is also provided, and Numerical experiments and examples are also presented. In Section 4, a note on how to deal with the situation 
when the time to expiry exceeds the provided radius of convergence is presented, followed by some concluding remarks given in the last section.

\section{The Heston-CIR hybrid model}

In this section, we will mainly discuss the specific model we adopt for European option pricing. Although the Black-Scholes model is very popular among market traders, some of the unrealistic assumptions made to achieve analytical tractability are inappropriate, such as the constant volatility assumption [8] and the constant interest rate assumption [1]. As a result, a number of modifications to the Black-Scholes model have been proposed to incorporate the effect of stochastic volatility and stochastic interest rate [6, 24]. One of the most popular models belonging to the category of stochastic volatility is the socalled Heston model, and there are mainly two reasons that can account for this. One is that the dynamics of the Heston model satisfy several properties, such as the non-negative property and the mean-reverting property being consistent with real market observations, and another is that there exists a closed-form pricing formula for European options, which makes it easy to be implemented in real markets. What we adopt here is actually a hybrid model combining the Heston stochastic volatility model and the CIR stochastic interest rate model, the dynamics of which under a risk-neutral measure $\mathbb{Q}$ are specified as

$$
\left[\begin{array}{c}
\frac{d S_{t}}{S_{t}} \\
d v_{t} \\
d r_{t}
\end{array}\right]=\mu^{Q} d t+\Sigma \times C \times\left[\begin{array}{l}
d W_{1, t} \\
d W_{2, t} \\
d W_{3, t}
\end{array}\right]
$$

where $S_{t}$ is the underlying price, $v_{t}$ is the volatility and $r_{t}$ is the risk-free interest rate. $W_{1, t}, W_{2, t}$ and $W_{3, t}$ are three Brownian motions that are independent with each other. The 
drift term $\mu^{Q}$ and the volatility term $\Sigma$ are defined as

$$
\mu^{Q}=\left[\begin{array}{c}
r_{t} \\
k\left(\theta-v_{t}\right) \\
\alpha\left(\beta-r_{t}\right)
\end{array}\right], \Sigma=\left[\begin{array}{ccc}
\sqrt{v} & 0 & 0 \\
0 & \sigma \sqrt{v} & 0 \\
0 & 0 & \eta \sqrt{r}
\end{array}\right]
$$

and $C$ is given as

$$
C=\left[\begin{array}{ccc}
1 & 0 & 0 \\
\rho & \sqrt{1-\rho^{2}} & 0 \\
0 & 0 & 1
\end{array}\right]
$$

such that the correlation matrix is

$$
C C^{T}=\left[\begin{array}{lll}
1 & \rho & 0 \\
\rho & 1 & 0 \\
0 & 0 & 1
\end{array}\right]
$$

After the introduction of the adopted Heston-CIR hybrid model, we are now ready to price European options, which is the main content of the next section.

\section{Pricing European options}

In this section, the problem of European option pricing is mainly considered under the adopted model. Specifically, a general pricing approach is firstly introduced for European option pricing based on the change of numeraire. Then, upon deriving an analytical formula for the characteristic function of the underlying price, we successfully obtain the closedform pricing formula in a series form, and a radius of convergence for such a series solution is also provided. Finally, numerical experiments are further carried out to show the accuracy as well as the convergence speed of our newly derived formula. 


\subsection{General pricing approach}

In the risk-neutral world, the price of a European option $U(S, v, r, t)$ is actually the expectation of the present value of the payoff, i.e.,

$$
U(S, v, r, t)=E^{Q}\left[e^{-\int_{t}^{T} r(s) d s} \max \left(S_{T}-K, 0\right) \mid S_{t}\right]
$$

with $K$ representing the strike price. Due to the existence of the stochastic interest rate, there are two different random variables in Equation (3.1), and it is very difficult to directly evaluate this expectation. Hence, in order to obtain the analytical expression of European

option prices, we here introduce a forward measure $\mathbb{Q}^{T}[6]$ such that Equation (3.1) can be rewritten as

$$
U(S, v, r, t)=P(r, t, T) E^{Q^{T}}\left[\max \left(S_{T}-K, 0\right) \mid S_{t}\right]
$$

where $P(r, t, T)$ is the price of a T-maturity zero coupon bond under $\mathbb{Q}$. This means that what we need to do first is to work out the formula for $P(r, t, T)$ as well as the dynamics of our model under the forward measure $\mathbb{Q}^{T}$. The following theorem presents the results of $P(r, t, T)$.

Theorem 1 If the risk-free interest rate follows the CIR model given in Dynamics (2.1), then the price of a T-maturity zero coupon bond $P(r, t, T)$ can be derived as

$$
P(r, t, T)=e^{A(t, T)-B(t, T) r}
$$

where $A(t, T)$ and $B(t, T)$ are

$$
\begin{aligned}
& \begin{aligned}
A(t, T) & =-\alpha \beta\left\{\frac{4}{(m-\alpha)(m+\alpha)} \ln \left[\frac{2 m+(m+\alpha)\left(e^{m(T-t)}-1\right)}{2 m}\right]+\frac{2}{\alpha-m}(T-t)\right\} \\
B(t, T) & =\frac{2\left(e^{\sqrt{m(T-t)}}-1\right)}{2 m+(\alpha+m)\left(e^{m(T-t)}-1\right)},
\end{aligned} \\
& \text { with } m=\sqrt{\alpha^{2}+2 \eta^{2}} \text {. }
\end{aligned}
$$


The proof of Theorem 1 is left in Appendix A.

After the derivation of $P(r, t, T)$, we are now ready to proceed to find the expression of the model dynamics under the forward measure $\mathbb{Q}^{T}$ with the implementation of the techniques illustrated in [6]. In particular, it should be noticed that the numeraire under $\mathbb{Q}$ is $N_{1, t}=e^{\int_{0}^{t} r(s) d s}$, and the numeraire under $\mathbb{Q}^{T}$ is $N_{2, t}=P(r, t, T)$. Thus, we can easily obtain

$$
d N_{1, t}=N_{1, t} r(t) d t
$$

which means that the volatility term of $N_{1, t}$ is $\sigma^{N_{1, t}}=(0,0,0)^{\prime}$ with $O^{\prime}$ denoting the transpose of the vector $O$. On the other hand, the dynamics of $N_{2, t}$ can be calculated as

$$
d N_{2, t}=N_{2, t}\left\{\left[\frac{d A}{d t}-r \frac{d B}{d t}-\alpha(\beta-r) B\right] d t-\eta \sqrt{r} B d W_{3, t}\right\}
$$

which implies that the volatility term of $N_{2, t}$ is $\sigma^{N_{2, t}}=\left(0,0,-\eta \sqrt{r} N_{2, t} B\right)^{\prime}$. As a result, according to the formula in [6], the drift term of the model dynamics under the forward measure $\mathbb{Q}^{T}$ can be obtained as

$$
\begin{aligned}
\mu^{Q_{T}}= & \mu^{Q}-\Sigma \times \rho \times\left(\frac{\sigma^{N_{1, t}}}{N_{1, t}}-\frac{\sigma^{N_{2, t}}}{N_{2, t}}\right) \\
= & {\left[\begin{array}{c}
r_{t} \\
k\left(\theta-v_{t}\right) \\
\alpha\left(\beta-r_{t}\right)
\end{array}\right]-\left[\begin{array}{ccc}
\sqrt{v} & 0 & 0 \\
0 & \sigma \sqrt{v} & 0 \\
0 & 0 & \eta \sqrt{r}
\end{array}\right] \times\left[\begin{array}{ccc}
1 & \rho & 0 \\
\rho & 1 & 0 \\
0 & 0 & 1
\end{array}\right] \times\left[\begin{array}{c}
0 \\
0 \\
\eta \sqrt{r} B
\end{array}\right] } \\
= & {\left[\begin{array}{c}
k\left(\theta-v_{t}\right) \\
\alpha \beta-\left[\alpha+B \eta^{2}\right] r
\end{array}\right] . }
\end{aligned}
$$


Clearly, the model dynamics under the forward measure $\mathbb{Q}^{T}$ can be expressed as

$$
\left[\begin{array}{c}
\frac{d S_{t}}{S_{t}} \\
d v_{t} \\
d r_{t}
\end{array}\right]=\left[\begin{array}{c}
r_{t} \\
k\left(\theta-v_{t}\right) \\
\alpha \beta-\left[\alpha+B \eta^{2}\right] r
\end{array}\right] d t+\Sigma \times C \times\left[\begin{array}{c}
d W_{1, t} \\
d W_{2, t} \\
d W_{3, t}
\end{array}\right]
$$

In the following subsection, a closed-form pricing formula for European options will be derived by calculating the expectation of the payoff function under the forward measure $\mathbb{Q}^{T}$ in Equation (3.2), based on the results obtained in this subsection.

\subsection{A closed-form pricing formula}

In this subsection, we present the specific procedures to find an analytical formula for European option prices under Dynamics (2.1).

According to the general pricing formula (3.2), if we make the transformation of $y=$ $\ln (S)$ and let $p(y)$ denote the probability density function of the underlying price, we can obtain the following expression of this formula

$$
U(y, v, r, t)=P(r, t, T)\left[P_{1}-K P_{2}\right]
$$

where $P_{1}=\int_{\ln K}^{+\infty} e^{y} p(y) d y$, and $P_{2}=\int_{\ln K}^{+\infty} p(y) d y$. Moreover, if we further assume that $f(\phi ; t, T, y, v, r)$ be the characteristic function of the underlying price, we can easily obtain

$$
P_{2}=\frac{1}{2}+\frac{1}{\pi} \int_{0}^{+\infty} \operatorname{Real}\left[\frac{e^{-j \phi \ln K} f(\phi ; t, T, y, v, r)}{j \phi}\right] d \phi
$$

where $j$ is the imaginary unit. On the other hand, it is not difficult to find that

$$
\int_{-\infty}^{+\infty} e^{y} p(y) d y=f(-j ; t, T, y, v, r)
$$


This implies that $\frac{e^{y} p(y)}{f(-j ; t, T, y, v, r)}$ is the density function of another random variable, whose characteristic function is actually $\frac{f(\phi-j ; t, T, y, v, r)}{f(-j ; t, T, y, v, r)}$. Therefore, $P_{1}$ can be derived as

$$
P_{1}=f(-j ; t, T, y, v, r)\left\{\frac{1}{2}+\frac{1}{\pi} \int_{0}^{+\infty} \operatorname{Real}\left[\frac{e^{-j \phi \ln K} f(\phi-j ; t, T, y, v, r)}{j \phi f(-j ; t, T, y, v, r)}\right] d \phi\right\}
$$

From Equation (3.8) and (3.9), it is obvious that in order to obtain the closed-form pricing formula, all we need to do is to figure out the analytical expression of the characteristic function under the forward measure $\mathbb{Q}^{T}$, the results of which is provided in the following theorem.

Theorem 2 If the underlying price follows the dynamics specified in Equation (3.6), the characteristic function of the underlying price $f(\phi ; \tau, y, v, r)$ can be derived as

$$
f=e^{C(\phi ; \tau)+D(\phi ; \tau) v+E(\phi ; \tau) r+j \phi y},
$$

where

$$
\begin{aligned}
D & =\frac{d-(j \phi \rho \sigma-k)}{\sigma^{2}} \cdot \frac{1-e^{d \tau}}{1-g e^{d \tau}}, \\
E & =-\frac{2 \sum_{n=0}^{+\infty}(n+1) \hat{a}_{n+1} \tau^{n}}{\eta^{2} \sum_{n=0}^{+\infty} \hat{a}_{n} \tau^{n}}, \\
C & =\frac{k \theta}{\sigma^{2}}\left\{[d-(j \phi \rho \sigma-k)] \tau-2 \ln \left(\frac{1-g e^{d \tau}}{1-g}\right)-\alpha \beta \int_{0}^{\tau} \frac{2 \sum_{n=0}^{+\infty}(n+1) \hat{a}_{n+1} t^{n}}{\eta^{2} \sum_{n=0}^{+\infty} \hat{a}_{n} t^{n}} d t\right\}, \\
d & =\sqrt{(j \phi \rho \sigma-k)^{2}+\sigma^{2}\left(j \phi+\phi^{2}\right)}, g=\frac{(j \phi \rho \sigma-k)-d}{(j \phi \rho \sigma-k)+d}, \\
\hat{I} & =2 \alpha m(n+1) \hat{a}_{n+1}+j \phi \eta^{2} m \hat{a}_{n}+(\alpha+m) \sum_{i=1}^{n}(n+2-i)(n+1-i) c_{i} \hat{a}_{n+2-i} \\
& =\left(\alpha^{2}+\alpha m+2 \eta^{2}\right) \sum_{i=1}^{n}(n+1-i) c_{i} \hat{a}_{n+1-i}+\frac{1}{2} j \phi \eta^{2}(\alpha+m) \sum_{i=1}^{n} c_{i} \hat{a}_{n-i},
\end{aligned}
$$


with $\tau=T-t$ and $c_{i}=\frac{m^{n}}{n !}$.

Proof. In order to derive Formula (3.10), we need to first know the definition of $f(\phi ; \tau, y, v, r)$, which is

$$
f(\phi ; \tau, y, v, r)=E^{Q^{T}}\left[e^{j \phi y_{T}} \mid y_{t}, v_{t}, r_{t}\right]
$$

After applying the Feynman-Kac theorem, we can easily find that $f(\phi ; \tau, y, v, r)$ should satisfy the following PDE

$$
\begin{aligned}
\frac{\partial f}{\partial \tau} & =\frac{1}{2} v \frac{\partial^{2} f}{\partial y^{2}}+\frac{1}{2} \sigma^{2} v \frac{\partial^{2} f}{\partial v^{2}}+\frac{1}{2} \eta^{2} r \frac{\partial^{2} f}{\partial r^{2}}+\rho \sigma v \frac{\partial^{2} f}{\partial y \partial v}+\left(r-\frac{1}{2} v\right) \frac{\partial f}{\partial y} \\
& +k(\theta-v) \frac{\partial f}{\partial v}+\left[\alpha \beta-\left(\alpha+B \eta^{2}\right) r\right] \frac{\partial f}{\partial r}
\end{aligned}
$$

with the terminal condition as

$$
\left.f\right|_{\tau=0}=e^{j \phi y}
$$

If we assume that $f(\phi ; \tau, y, v, r)$ takes the form of

$$
f=e^{C(\phi ; \tau)+D(\phi ; \tau) v+E(\phi ; \tau) r+j \phi y},
$$

and substitute it into the original PDE (3.12), we can further obtain the following three ODEs (ordinary differential equations)

$$
\begin{aligned}
& \frac{d D}{d \tau}=\frac{1}{2} \sigma^{2} D^{2}+(j \phi \rho \sigma-k) D-\frac{1}{2}\left(j \phi+\phi^{2}\right), \\
& \frac{d E}{d \tau}=\frac{1}{2} \eta^{2} E^{2}-\left[\alpha+B(\tau) \eta^{2}\right] E+j \phi, \\
& \frac{d C}{d \tau}=k \theta D+\alpha \beta E,
\end{aligned}
$$

with $D(\phi ; 0)=E(\phi ; 0)=C(\phi ; 0)=0$. Obviously, the ODE governing $D(\phi ; \tau)$ is actually a Riccati equation with constant coefficients, which means that it can be easily solved with 
some simple algebraic calculations. In contrast, although the ODE governing $E(\phi ; \tau)$ is again a Riccati equation, it is very difficult to work out the expression of $E(\phi ; \tau)$ as there exists a time-dependent coefficient. Fortunately, we finally come out one way, the first step of which is to make the transform of

$$
E=-\frac{2 u^{\prime}}{\eta^{2} u}
$$

and substitute it into the original ODE so that we can obtain

$$
u^{\prime \prime}+\left[\alpha+B(\tau) \eta^{2}\right] u^{\prime}+\frac{1}{2} j \phi \eta^{2}=0
$$

We actually seek a series solution to Equation (3.15), which is expressed as

$$
u=\sum_{n=0}^{+\infty} a_{n} \tau^{n}
$$

Substituting Equation (3.16) into (3.15) can yield

$$
\begin{aligned}
& {\left[2 m+(\alpha+m)\left(e^{m \tau}-1\right)\right] \sum_{n=0}^{+\infty}(n+1)(n+2) a_{n+2} \tau^{n}+\frac{1}{2} j \phi \eta^{2}\left[2 m+(\alpha+m)\left(e^{m \tau}-1\right)\right] \sum_{n=0}^{+\infty} a_{n} \tau^{n}} \\
& +\left\{\alpha\left[2 m+(\alpha+m)\left(e^{m \tau}-1\right)\right]+2 \eta^{2}\left(e^{m \tau}-1\right)\right\} \sum_{n=0}^{+\infty}(n+1) a_{n+1} \tau^{n}=0 .
\end{aligned}
$$

As $e^{m \tau}$ can be expanded as $e^{m \tau}=\sum_{n=0}^{+\infty} c_{n} \tau^{n}$, Equation (3.17) can be rearranged as

$$
\begin{aligned}
& {\left[2 m+(\alpha+m) \sum_{n=1}^{+\infty} c_{n} \tau^{n}\right] \sum_{n=0}^{+\infty}(n+1)(n+2) a_{n+2} \tau^{n}+\left[j \phi \eta^{2} m+\frac{1}{2} j \phi \eta^{2}(\alpha+m) \sum_{n=1}^{+\infty} c_{n} \tau^{n}\right] \sum_{n=0}^{+\infty} a_{n} \tau^{n}} \\
& +\left[2 \alpha m+\left(\alpha^{2}+m \alpha+2 \eta^{2}\right) \sum_{n=1}^{+\infty} c_{n} \tau^{n}\right] \sum_{n=0}^{+\infty}(n+1) a_{n+1} \tau^{n}=0
\end{aligned}
$$

Since Equation (3.18) should hold for any $\tau$, the coefficient of $\tau^{n}$ should be zero for all 
$n \geq 0$. Therefore, what we can obtain is

$$
\begin{aligned}
& 2 m(n+1)(n+2) a_{n+2}+2 \alpha m(n+1) a_{n+1}+j \phi \eta^{2} m a_{n}+(\alpha+m) \sum_{i=1}^{n}(n+2-i)(n+1-i) c_{i} a_{n+2-i} \\
& +\left(\alpha^{2}+\alpha m+2 \eta^{2}\right) \sum_{i=1}^{n}(n+1-i) c_{i} a_{n+1-i}+\frac{1}{2} j \phi \eta^{2}(\alpha+m) \sum_{i=1}^{n} c_{i} a_{n-i}=0
\end{aligned}
$$

from which the expression of $a_{n+2}$ can be written as

$$
a_{n+2}=-\frac{I}{2 m(n+1)(n+2)}
$$

where

$$
\begin{aligned}
I & =2 \alpha m(n+1) a_{n+1}+j \phi \eta^{2} m a_{n}+(\alpha+m) \sum_{i=1}^{n}(n+2-i)(n+1-i) c_{i} a_{n+2-i} \\
& +\left(\alpha^{2}+\alpha m+2 \eta^{2}\right) \sum_{i=1}^{n}(n+1-i) c_{i} a_{n+1-i}+\frac{1}{2} j \phi \eta^{2}(\alpha+m) \sum_{i=1}^{n} c_{i} a_{n-i} .
\end{aligned}
$$

Obviously, if we can obtain the value of $a_{0}$ and $a_{1}$, all the coefficient $a_{n}, n \geq 2$ can be obtained from Equation (3.20). Unfortunately, from the condition $E(\phi ; 0)=0$, we can only derive $a_{1}=0$, and it seems it is impossible to find out $a_{0}$, which implies that our task has not been finished. Finally, we come out an alternative way and introduce a new coefficient defined as $\hat{a}_{n}=\frac{a_{n}}{a_{0}}$. In this case, $E(\phi ; \tau)$ can be further expressed as

$$
E=-\frac{2 \sum_{n=0}^{+\infty}(n+1) \hat{a}_{n+1} \tau^{n}}{\eta^{2} \sum_{n=0}^{+\infty} \hat{a}_{n} \tau^{n}}
$$

according to Equation (3.14). On the other hand, we also have

$$
\hat{a}_{n+2}=-\frac{\hat{I}}{2 m(n+1)(n+2)},
$$

with $\hat{I}=\frac{I}{a_{0}}$. In this situation, we can easily obtain $\hat{a}_{0}=1$ and $\hat{a}_{1}=0$, which implies that 
all the coefficients can be found with Equation (3.22), and thus we have already derived the formula for $E(\phi ; \tau)$. Finally, $C(\phi ; \tau)$ can be worked out with direct integration. This has completed the proof.

Obviously, what we present here is actually a series solution, and thus our solution can never be regarded as "closed-form" if there is no convergence of proof. Although it seems very difficult to theoretically prove the convergence of the characteristic function due to the complicatedness of its expression, we fortunately find an alternative approach. It should be pointed out that the first time we introduce the series solution is in Equation (3.15), and thus the characteristic function will be regarded as converged if this series solution converges. Therefore, a radius of convergence for the series solution is presented in the following theorem.

Theorem 3 The series solution $u=\sum_{n=0}^{+\infty} a_{n} \tau^{n}$ will always converge if

$$
\tau \leq \frac{1}{m} \sqrt{\left[\ln \left(\frac{m-\alpha}{m+\alpha}\right)\right]^{2}+\pi^{2}}
$$

The proof of Theorem 3 is left in Appendix B.

Obviously, by now we have successfully derived a closed-form pricing formula for European options in a series form under the Heston-CIR hybrid model, which is Formula (3.7) with $P(r, t, T), P_{1}$ and $P_{2}$ given in Equation (3.3), (3.9) and (3.8) respectively. However, there are two remaining issues; one is that we should examine its convergence speed as this is also an important issue in reality, given the recent trend of algorithmic trading, and another is that we still need to check the validity of the newly derived formula to ensure there are no algebraic errors in our calculation. These issues will be further discussed in the following subsection. 


\subsection{Numerical experiments and discussions}

In this subsection, the speed of convergence for our pricing formula is numerically checked with the parameters chosen under the restriction (3.23), and then we verify our newly derived formula by comparing the results calculated with our formula and those obtained from Monte Carlo simulation. Afterwards, the difference between our adopted Heston-CIR hybrid model and the standard Heston model is shown to demonstrate the influence of introducing stochastic interest rate. In the following, unless otherwise stated, parameters we use are listed as follows. The mean-reverting speed $k$, the long-term mean $\theta$ and the volatility of volatility $\sigma$ take the value of $10,0.2$ and 0.1 respectively, while the corresponding parameters for the stochastic interest rate satisfy $\alpha=2, \beta=0.05$ and $\eta=0.05$. Moreover, the initial value for the volatility $v_{0}$ and the risk-free interest rate $r_{0}$ equal to 0.05 and 0.03 respectively. The strike price $K$ for the option is set to be 100 , the underlying price $S$ is 100 , and the time to expiry $\tau$ is 1 .

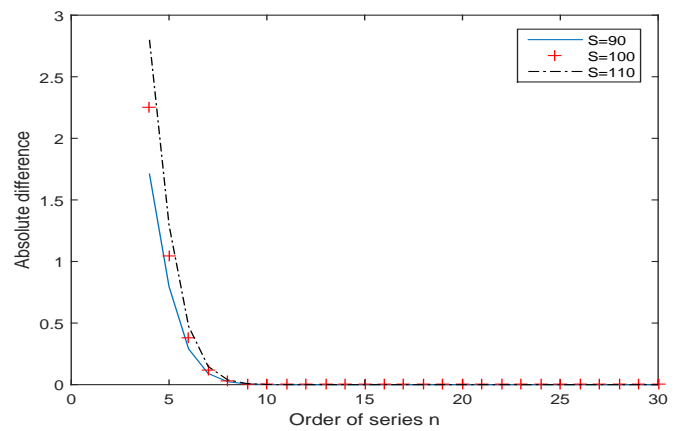

(a) Absolute difference between the $(n+1)$-term price and $n$-term price.

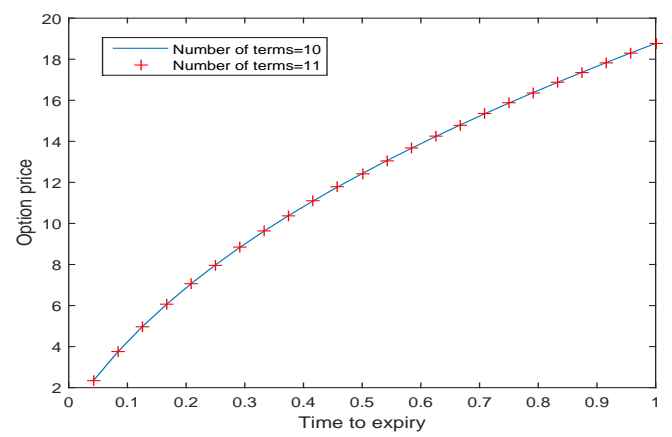

(b) The 10-term and 11-term option price.

Figure 1: The convergence of our solution.

In Figure 1, our solution taking different number of terms is numerically shown to demonstrate the speed of convergence. Specifically, depicted in Figure 1(a) is the absolute difference between the $(n+1)$-term price and $n$-term price against the used order of the series $n$. It should be pointed out that our solution can be viewed as converged if the absolute difference becomes zero when the order of series reaches a critical point, and it remains 
zero from then on, no matter how large the order of series is. Thus, the phenomenon that the absolute difference decreases very sharply to zero when we increase the order of series clearly demonstrates that our solution converges very rapidly, and we only need to use a few terms to obtain a converged solution under the chosen set of parameters. To exhibit this more clearly, we present the 10-term and 11-term option price in Figure 1(b), with the absolute difference being in the order of $10^{-4}$. This can certainly show that the 10 -term price can be regarded as the converged option price.

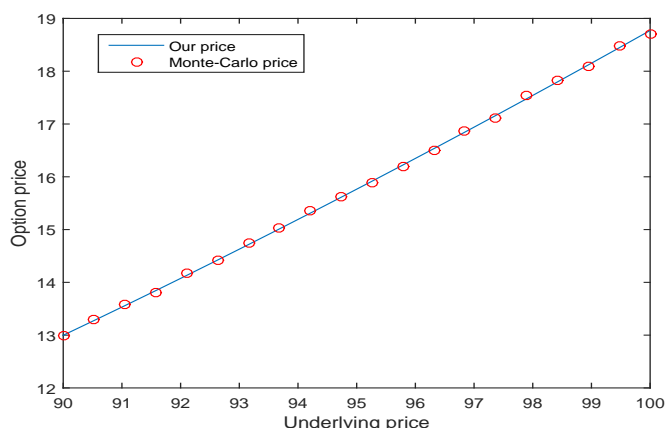

(a) Our price vs Monte-Carlo price.

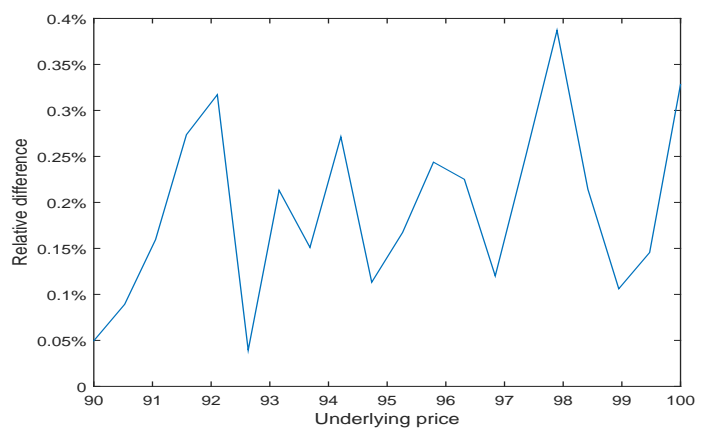

(b) The relative difference between our price and Monte-Carlo price.

Figure 2: The comparison of option prices calculated with our formula and those obtained through Monte Carlo simulation.

Apart from the convergence speed, another issue that needs to be addressed is the accuracy of our newly derived formula so that we can ensure there are no algebraic errors. Therefore, what is presented in Figure 2 is the numerical comparison of option prices from our formula and those from Monte Carlo simulation. In specific, what can be observed first in Figure 2(a) is that option prices are an monotonic increasing function of the underlying price, which is expected since a higher underlying price will potentially lead to a higher payoff of an European call option. Also, our price is quite close to the Monte-Carlo price in the point-wise manner, no matter the option is in-the-money, at-the-money or out-ofthe-money. To further demonstrate the accuracy of our formula, the relative difference between our price and Monte-Carlo price is provided in Figure 2(b). With the maximum relative difference being less than $0.4 \%$, we can certainly reach the conclusion that our 
formula is correct and can be used in real markets.

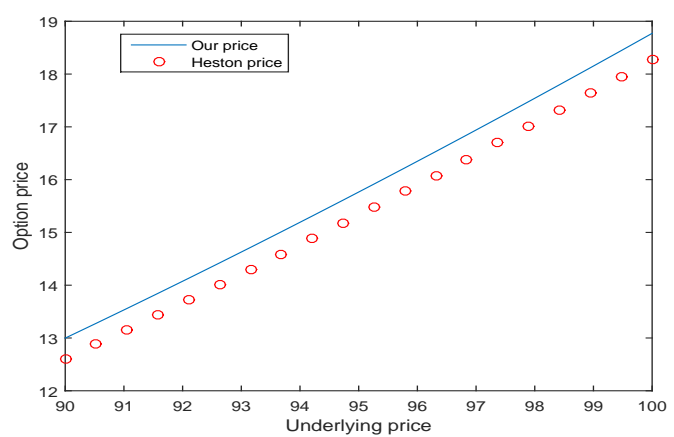

(a) Our price and the Heston price with respect to the underlying price.

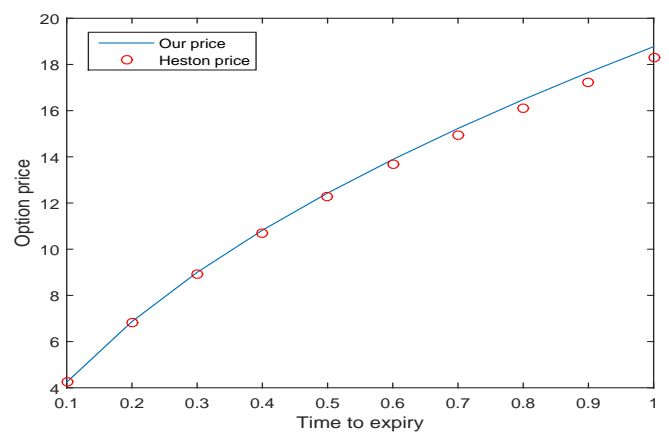

(b) Our price and the Heston price with respect to the time to expiry.

Figure 3: The comparison of option prices under our hybrid Heston-CIR model and the standard Heston model.

By now we are confident of our newly derived pricing formula and thus we can study the influence of introducing stochastic interest rate into the Heston model, which is presented in Figure 3. In particular, what are shown in Figure 3(a) are option prices under our model and the Heston model with the corresponding parameters in the two models being the same. It can be clearly observed that our price is higher than the Heston price under the chosen set of parameters, and these prices under the two models are almost parallel to each other. On the other hand, a different pattern can be witnessed in Figure 3(b), where option prices are shown with different time to expiry. Obviously, when the time to expiry is very small, our price and the Heston price are almost the same, and when the time to expiry becomes larger, the difference between our price and the Heston price is enlarged. This is reasonable as the stochastic interest rate will not change distinctly if the time to expiry is short, while the increase in the time to expiry means there are longer time for the variation of the interest rate and thus will certainly contribute to the larger change of the interest rate, which can account for the widened gap.

It should be noted that the above discussion focuses on the situation when the time to expiry is smaller than the derived radius of convergence. However, we still do not known how to price options when the time to expiry exceeds this specific value. This issue will be 
illustrated in the following section.

\section{$4 \quad$ A note on $\tau>\frac{1}{m} \sqrt{\left[\ln \left(\frac{m-\alpha}{m+\alpha}\right)\right]^{2}+\pi^{2}}$}

In this section, we shall briefly discuss what we need to do if the time to expiry is not within the converged area, i.e., $\tau>\frac{1}{m} \sqrt{\left[\ln \left(\frac{m-\alpha}{m+\alpha}\right)\right]^{2}+\pi^{2}}$. In this case, the solution derived in Section 3 can not be used as there is no guarantee of convergence, and thus alternative solutions should be found.

In particular, we take a same pricing procedure to firstly arrive at Equation (3.15). Then, if we let

$$
t_{l}=\sum_{i=1}^{l} \frac{1}{m} \sqrt{\left[\ln \left(\frac{m-\alpha}{m+\alpha}\right)\right]^{2}+\pi^{2}} l+\frac{1}{m} \ln \left(\frac{m-\alpha}{m+\alpha}\right),
$$

we can expand the solution to $\operatorname{ODE}(3.15)$ at the point $\tau=t_{l}$ when $t_{l} \leq \tau \leq t_{l+1}$ as

$$
u=\sum_{n=0}^{+\infty} a_{n}^{(l)}\left(\tau-t_{l}\right)^{2}
$$

If we further expand $e^{m \tau}$ as

$$
e^{m \tau}=e^{m t_{l}} \sum_{n=0}^{+\infty} c_{n}\left(\tau-t_{l}\right)^{n}
$$

the original ODE can be rearranged as

$$
\begin{aligned}
& {\left[2 m+(m+\alpha)\left(e^{m t_{l}}-1\right)+(\alpha+m) e^{m t_{l}} \sum_{n=1}^{+\infty} c_{n}\left(\tau-t_{l}\right)^{n}\right] \sum_{n=0}^{+\infty}(n+1)(n+2) a_{n+2}^{(l)}\left(\tau-t_{l}\right)^{n}} \\
& {\left[2 \alpha m+\left(\alpha^{2}+\alpha m+2 \eta^{2}\right)\left(e^{m t_{l}}-1\right)+\left(\alpha^{2}+\alpha m+2 \eta^{2}\right) e^{m t_{l}} \sum_{n=1}^{+\infty} c_{n}\left(\tau-t_{l}\right)^{n}\right] \sum_{n=0}^{+\infty}(n+1) a_{n+1}^{(l)}\left(\tau-t_{l}\right)^{n}} \\
& \frac{1}{2} j \phi \eta^{2}\left[2 m+(\alpha+m)\left(e^{m t_{l}}-1\right)+(\alpha+m) e^{m t_{l}} \sum_{n=1}^{+\infty} c_{n}\left(\tau-t_{l}\right)^{n}\right] \sum_{n=0}^{+\infty} a_{n}^{(l)}\left(\tau-t_{l}\right)^{n}
\end{aligned}
$$

It should be noted that Equation (4.2) should hold for any $\tau$, which means that the 
coefficients of $\left(\tau-t_{l}\right)^{k}, k \geq 0$ should equal to zero. In this case, we can easily obtain

$$
\left[2 m+(m+\alpha)\left(e^{m t_{l}}-1\right)\right](n+1)(n+2) a_{n+2}^{(l)}+I^{(l)}=0,
$$

where $I^{(l)}$ is defined as

$$
\begin{aligned}
I^{(l)} & =\left[2 \alpha m+\left(\alpha^{2}+\alpha m+2 \eta^{2}\right)\left(e^{m t_{l}}-1\right)\right](n+1) a_{n+1}^{(l)}+\frac{1}{2} j \phi \eta^{2}\left[2 m+(\alpha+m)\left(e^{m t_{l}}-1\right)\right] a_{n}^{(l)} \\
& +\left(\alpha^{2}+\alpha m+2 \eta^{2}\right) e^{m t_{l}} \sum_{i=1}^{n}(n+1-i) c_{i} a_{n+1-i}^{(l)}+(\alpha+m) e^{m t_{l}} \sum_{i=1}^{n}(n+2-i)(n+1-i) c_{i} a_{n+2-i}^{(l)} \\
& +\frac{1}{2} j \phi \eta^{2}(\alpha+m) e^{m t_{l}} \sum_{i=1}^{n} c_{i} a_{n-i}^{(l)} .
\end{aligned}
$$

This can further yield

$$
a_{n+2}^{(l)}=-\frac{I^{(l)}}{\left[2 m+(m+\alpha)\left(e^{m t_{l}}-1\right)\right](n+1)(n+2)} .
$$

Now, we have met a same problem that only $a_{1}^{(l)}=0$ can be worked out while $a_{0}^{(l)}$ remains unknown. Thus we take a similar technique, introducing $\hat{a}_{n}^{(l)}=\frac{a_{n}^{(l)}}{a_{0}^{(l)}}$, by doing which

$$
\hat{a}_{n+2}^{(l)}=-\frac{\hat{I}^{(l)}}{\left[2 m+(m+\alpha)\left(e^{m t_{l}}-1\right)\right](n+1)(n+2)},
$$

with $\hat{I}^{(l)}=\frac{I}{a_{0}^{(l)}}, \hat{a}_{0}^{(l)}=1$ and $\hat{a}_{1}^{(l)}=0$. Clearly, we have now derived a set of analytical pricing formulae for European options, which are in the same form of Equation (3.7) except $u$ and $\hat{a}_{n}, n \geq 0$ are now replaced by Equation (4.1) and (4.5) respectively.

Another issue that should be addressed is that whether each pricing formula in this set will converge on their defined domain so that we can always find a converged solution for any $\tau \in[0,+\infty]$. This means that for any positive number $l$, we need to prove that the solution expanded at the point $\tau=t_{l}$ is converged on $t_{l} \leq \tau \leq t_{l+1}$. According to the proof 
for Theorem 3, we can easily show that all the singularities of ODE (3.15) are

$$
\frac{1}{m} \ln \left(\frac{m-\alpha}{m+\alpha}\right)+j \frac{(2 k+1) \pi}{m}, k=0,1,2 \ldots
$$

which means that the nearest singularity to $\tau=t_{l}$ is $\frac{1}{m} \ln \left(\frac{m-\alpha}{m+\alpha}\right)+j \frac{\pi}{m}$. Thus, a radius of convergence for the solution is

$$
\begin{aligned}
R_{l} & =\sqrt{\frac{1}{m^{2}}\left\{\sum_{i=1}^{l} \sqrt{\left[\ln \left(\frac{m-\alpha}{m+\alpha}\right)\right]^{2}+\pi^{2} l}\right\}^{2}+\frac{\pi^{2}}{m^{2}}} \\
& >\sqrt{\frac{1}{m^{2}}\left[\ln \left(\frac{m-\alpha}{m+\alpha}\right)^{2}+\pi^{2} l\right]} \\
& =\frac{1}{m} \sqrt{\ln \left(\frac{m-\alpha}{m+\alpha}\right)^{2}+\pi^{2}(l+1)} \\
& =t_{l+1}-t_{l},
\end{aligned}
$$

from which we can certainly conclude that the solution expanded at the point $\tau=t_{l}$ converges on $\left[t_{l}, t_{l+1}\right]$. Therefore, for any $\tau \in\left[t_{1},+\infty\right]$, we can always find a converged solution. Hence, the only left interval is $\left[0, t_{1}\right]$ and what we need to find is a converged solution on this particular region. In fact, due to the fact $\alpha>0, m>0$, we can easily deduce that $\frac{1}{m} \ln \left(\frac{m-\alpha}{m+\alpha}\right)<0$, which can further yield

$$
t_{1}=\frac{1}{m} \sqrt{\left[\ln \left(\frac{m-\alpha}{m+\alpha}\right)\right]^{2}+\pi^{2}}+\frac{1}{m} \ln \left(\frac{m-\alpha}{m+\alpha}\right)<\frac{1}{m} \sqrt{\left[\ln \left(\frac{m-\alpha}{m+\alpha}\right)\right]^{2}+\pi^{2}} .
$$

As a result, the solution derived in Section 3 converges when $\tau \in\left[0, t_{1}\right]$, and combining all the discussion above, it is not difficult to find that no matter what the value of $\tau$ is, we are always able to find a converged solution. 


\section{Conclusion}

In this paper, the underlying price is assumed to follow the Heston stochastic volatility model with the constant interest rate replaced by the CIR stochastic interest rate. With the technique of the numeraire change, we present a closed-form pricing formula for European options, which is in an infinite series form. Moreover, we have provided the radius of convergence for this solution, supplemented by the results of numerical experiments, demonstrating that our solution indeed converges very rapidly.

\section{References}

[1] M. Abudy and Y. Izhakian. Pricing stock options with stochastic interest rate. International Journal of Portfolio Analysis and Management, 1(3):250-277, 2013.

[2] G. Bakshi, C. Cao, and Z. Chen. Empirical performance of alternative option pricing models. The Journal of Finance, 52(5):2003-2049, 1997.

[3] S. Beckers. Variances of security price returns based on high, low, and closing prices. Journal of Business, pages 97-112, 1983.

[4] C. M. Bender and S. A. Orszag. Advanced mathematical methods for scientists and engineers I. Springer Science \& Business Media, 1999.

[5] F. Black and M. Scholes. The pricing of options and corporate liabilities. The Journal of Political Economy, pages 637-654, 1973.

[6] D. Brigo and F. Mercurio. Interest rate models-theory and practice: with smile, inflation and credit. Springer Science \& Business Media, 2007.

[7] E. Derman and I. Kani. Riding on a smile. Risk, 7(2):32-39, 1994. 
[8] B. Dumas, J. Fleming, and R. E. Whaley. Implied volatility functions: Empirical tests. The Journal of Finance, 53(6):2059-2106, 1998.

[9] B. Dupire et al. Pricing with a smile. Risk, 7(1):18-20, 1994.

[10] M. Forde and A. Jacquier. Robust approximations for pricing Asian options and volatility swaps under stochastic volatility. Applied Mathematical Finance, 17(3):241$259,2010$.

[11] L. A. Grzelak and C. W. Oosterlee. On the Heston model with stochastic interest rates. SIAM Journal on Financial Mathematics, 2(1):255-286, 2011.

[12] L. A. Grzelak, C. W. Oosterlee, and S. Van Weeren. Extension of stochastic volatility equity models with the Hull-White interest rate process. Quantitative Finance, 12(1):89-105, 2012.

[13] P. S. Hagan, D. Kumar, A. S. Lesniewski, and D. E. Woodward. Managing smile risk. 70+ DVDs FOR SALE \& EXCHANGE, page 249, 2002.

[14] X.-J. He and S.-P. Zhu. An analytical approximation formula for European option pricing under a new stochastic volatility model with regime-switching. Journal of Economic Dynamics and Control, 71:77-85, 2016.

[15] X.-J. He and S.-P. Zhu. Pricing European options with stochastic volatility under the minimal entropy martingale measure. European Journal of Applied Mathematics, 27(02):233-247, 2016.

[16] S. L. Heston. A closed-form solution for options with stochastic volatility with applications to bond and currency options. Review of Financial Studies, 6(2):327-343, 1993.

[17] J. Hull and A. White. The pricing of options on assets with stochastic volatilities. The Journal of Finance, 42(2):281-300, 1987. 
[18] J. C. Hull and A. D. White. Using Hull-White interest rate trees. the Journal of Derivatives, 3(3):26-36, 1996.

[19] H. Johnson and D. Shanno. Option pricing when the variance is changing. Journal of Financial and Quantitative Analysis, 22(02):143-151, 1987.

[20] K. Rindell. Pricing of index options when interest rates are stochastic: An empirical test. Journal of Banking \& Finance, 19(5):785-802, 1995.

[21] M. Rubinstein. Implied binomial trees. The Journal of Finance, 49(3):771-818, 1994.

[22] R. Schöbel and J. Zhu. Stochastic volatility with an Ornstein-Uhlenbeck process: an extension. European Finance Review, 3(1):23-46, 1999.

[23] L. O. Scott. Option pricing when the variance changes randomly: Theory, estimation, and an application. Journal of Financial and Quantitative Analysis, 22(04):419-438, 1987.

[24] E. M. Stein and J. C. Stein. Stock price distributions with stochastic volatility: An analytic approach. Review of Financial Studies, 4(4):727-752, 1991.

[25] J. B. Wiggins. Option values under stochastic volatility: Theory and empirical estimates. Journal of Financial Economics, 19(2):351-372, 1987.

[26] S.-P. Zhu and X. He. On the convergence of he and zhu's new series solution for pricing options with the heston model. Acta Mathematica Universitatis Comenianae, pages 1-7, 2017.

\section{Appendix A}

Here is the solution procedure to deriver the expression of $P(r, t, T)$. According to the risk-neutral pricing rule that the discounted asset price should be a martingale, we can 
easily find that $P(r, t, T)$ should satisfy the following PDE system

$$
\left\{\begin{array}{l}
\frac{\partial P}{\partial t}+\alpha(\beta-r) \frac{\partial P}{\partial r}+\frac{1}{2} \eta^{2} r \frac{\partial^{2} P}{\partial r^{2}}-r P=0 \\
\left.P(r, t, T)\right|_{t=T}=1
\end{array}\right.
$$

If we assume that $P(r, t, T)$ takes the form of

$$
P(r, t, T)=e^{A(t, T)-B(t, T) r}
$$

and substitute it into the pricing PDE $(A-1)$, we can obtain the following two ODEs

$$
\left\{\begin{array}{l}
\frac{d B}{d t}=\frac{1}{2} \eta^{2} B^{2}+\alpha B-1, \quad B(T, T)=0 \\
\frac{d A}{d t}=\alpha \beta B, \quad A(T, T)=0
\end{array}\right.
$$

The equation governing $B(t, T)$ is actually a Riccati equation, which can be easily solved with some algebraic calculation. The expression of $A(t, T)$ can then be obtained by direct integration. This has completed the proof.

\section{Appendix B}

Here is the complete proof for the radius of convergence. According to one existing theorem, it is not difficult to find that the radius of convergence of the series solution to a second order linear ODE expanded at an ordinary point is at least as large as the distance between the ordinary point to the nearest singularity of the ODE [4]. Therefore, what we need to do is to find the nearest singularity to zero.

Specifically, $\frac{1}{2} j \phi \eta^{2}$ in Equation (3.15) is actually a constant and thus analytic in the 
entire complex domain. Moreover, if we denote

$$
\begin{aligned}
& F_{1}(\tau)=2 m \alpha+\left(\alpha^{2}+m \alpha+2 \eta^{2}\right)\left(e^{m \tau}-1\right) \\
& F_{2}(\tau)=2 m+(\alpha+m)\left(e^{m \tau}-1\right)
\end{aligned}
$$

it is not difficult to find that $\alpha+B(\tau) \eta^{2}=\frac{F_{1}(\tau)}{F_{2}(\tau)}$. Considering the fact that $F_{1}(\tau)$ and $F_{2}(\tau)$ are both analytic in the complex domain, all the singularities can be obtained if we set $F_{2}(\tau)=0$, the results of which are given as

$$
\tau_{k}=\frac{1}{m} \ln \left(\frac{m-\alpha}{m+\alpha}\right)+j \frac{(2 k+1) \pi}{m}, k=0,1,2 \ldots
$$

This means that the nearest singularity to zero is $\frac{1}{m} \ln \left(\frac{m-\alpha}{m+\alpha}\right)+j \frac{\pi}{m}$, and thus the radius of convergence is at least $\frac{1}{m} \sqrt{\left[\ln \left(\frac{m-\alpha}{m+\alpha}\right)\right]^{2}+\pi^{2}}$, which has completed the proof. 